\title{
Involvement of Rho-kinase in sphingosine 1-phosphate-stimulated HSP27 induction in osteoblasts
}

\author{
CHIHO MINAMITANI ${ }^{1,2}$, TAKANOBU OTSUKA ${ }^{1}$, SHINJI TAKAI ${ }^{2}$, RIE MATSUSHIMA-NISHIWAKI ${ }^{2}$, \\ SEIJI ADACHI ${ }^{2}$, YOSHITERU HANAI ${ }^{2}$, JUN MIZUTANI ${ }^{1}$, HARUHIKO TOKUDA $^{2,3}$ and OSAMU KOZAWA ${ }^{2}$ \\ ${ }^{1}$ Department of Orthopedic Surgery, Nagoya City University Graduate School of Medical Sciences, Nagoya 467-8601; \\ ${ }^{2}$ Department of Pharmacology, Gifu University Graduate School of Medicine, Gifu 501-1194; \\ ${ }^{3}$ Department of Clinical Laboratory, National Hospital for Geriatric Medicine, \\ National Center for Geriatrics and Gerontology, Obu 474-8511, Japan
}

Received November 28, 2008; Accepted February 2, 2009

DOI: 10.3892/ijmm_00000209

\begin{abstract}
We previously reported that sphingosine 1-phosphate induces heat shock protein 27 (HSP 27) via activation of phosphatidylinositol 3-kinase (PI3K)/Akt and p38 mitogenactivated protein (MAP) kinase in osteoblast-like MC3T3-E1 cells. In the present study, we investigated whether Rho-kinase is implicated in sphingosine 1-phosphate-stimulated induction of HSP27 in MC3T3-E1 cells. Sphingosine 1-phosphate timedependently induced the phosphorylation of myosin phosphatase targeting subunit (MYPT-1), a Rho-kinase substrate. Y27632, a specific Rho-kinase inhibitor, significantly reduced sphingosine 1-phosphate-stimulated HSP27 induction, as well as MYPT-1 phosphorylation. Fasudil, another inhibitor of Rhokinase, also suppressed sphingosine 1-phosphate-stimulated HSP27 induction. Y27632, as well as fasudil, attenuated sphingosine 1-phosphate-induced phosphorylation of p38 MAP kinase. However, Akt phosphorylation induced by sphingosine 1-phosphate was not affected by either Rho-kinase inhibitor. These results strongly suggest that Rho-kinase regulates sphingosine 1-phosphate-stimulated induction of HSP27 at a point upstream of p38 MAP kinase in osteoblasts.
\end{abstract}

\section{Introduction}

Sphingosine 1-phosphate is a metabolite of sphingomyelin. It is generally recognized that sphingomyelin is catalyzed by sphingomyelinase, resulting in the formation of ceramide, which is subsequently metabolized to sphingosine and sphingosine

Correspondence to: Dr Osamu Kozawa, Department of Pharmacology, Gifu University Graduate School of Medicine, Gifu 501-1194, Japan

E-mail: okozawa@gifu-u.ac.jp

Key words: mitogen-activated protein kinase, heat shock protein, MC3T3-E1 cells 1-phosphate (1). Accumulating evidence indicates that sphingosine 1-phosphate plays an important role in essential cellular functions such as proliferation, differentiation, and migration (1-3). In our study (4), we previously reported that sphingosine 1-phosphate stimulates interleukin-6 (IL-6) synthesis in osteoblast-like MC3T3-E1 cells. However, the exact mechanism of sphingosine 1-phosphate in bone metabolism is not yet clarified.

Heat shock proteins (HSPs) are expressed in both prokaryotic and eukaryotic cells in response to biological stress, such as heat and chemical stress (5). HSPs are classified into high- and low-molecular-weight HSPs based on apparent molecular size. Low-molecular-weight HSPs with a molecular mass from 10 to $30 \mathrm{kDa}$, such as HSP27 and $\alpha \mathrm{B}$-crystallin, have high homology in amino acid sequences $(6,7)$. Though the functions of low-molecular-weight HSPs are lesser known than those of high-molecular-weight HSPs, it is generally accepted that they have chaperoning functions like highmolecular-weight HSPs $(6,7)$. HSP27 is rapidly phosphorylated in response to various stresses, as well as exposure to cytokines and mitogens $(8,9)$. Under unstimulated conditions, HSP27 exists as a high-molecular weight aggregated form. It is rapidly dissociated as a result of phosphorylation $(10,11)$. The phosphorylation-induced dissociation from the aggregated form correlates with the loss of molecular chaperone activity $(10,11)$. In our previous studies $(12,13)$, we showed that sphingosine 1-phosphate stimulates the induction of HSP27 via p38 mitogen-activated protein (MAP) kinase activation and phosphatidylinositol 3-kinase (PI3K)/Akt activation in osteoblast-like MC3T3-E1 cells.

Previous studies suggest that Rho and the downstream effector, Rho-associated kinase (Rho-kinase), play crucial roles in a variety of cellular functions, such as cell motility and smooth muscle contraction (14-16). Regarding bone metabolism, it was shown that the Rho/Rho-kinase pathway stimulates osteoblast proliferation whereas it inhibits osteoblast differentiation (17). In addition, we recently showed that Rhokinase regulates prostaglandin F2 $\alpha$-stimulated IL- 6 synthesis through p38 MAP kinase activation in MC3T3-E1 cells (18). However, the exact role of Rho-kinase in osteoblasts is not yet elucidated. 
In the present study, we further investigated whether Rhokinase is involved in sphingosine 1-phosphate-stimulated induction of HSP27 in osteoblast-like MC3T3-E1 cells. We show that Rho-kinase regulates sphingosine 1-phosphatestimulated induction of HSP 27 via p38 MAP kinase activation, but not via the PI3K/Akt pathway in these cells.

\section{Materials and methods}

Materials. Sphingosine 1-phosphate and hydroxyfasudil (fasudil) were purchased from Sigma Chemical Co. (St. Louis, MO, USA). Y27632 was obtained from Merk KGaA (Darmstadt, Germany). Phospho-specific myosin phosphatase targeting subunit (MYPT)-1 antibodies were purchased from Millipore, Co. (Billerica, MA, USA). MYPT-1 and glyceraldehyde 3-phosphate dehydrogenase (GAPDH) antibodies were obtained from Santa Cruz Biotechnology, Inc. (Santa Cruz, CA, USA). Antibodies for phospho-specific p38 MAP kinase, p38 MAP kinase, phospho-specific Akt and Akt were purchased from Cell Signaling Technology, Inc. (Beverly, MA, USA). HSP 27 antibodies were obtained from R\&D Systems Inc. (Minneapolis, MN, USA). The ECL Western blotting detection system was purchased from Amersham Biosciences (Piscataway, NJ, USA). Other materials and chemicals were obtained from commercial sources. Sphingosine 1-phosphate and Y27632 were dissolved in dimethyl sulfoxide (DMSO). The maximum concentration of DMSO was $0.1 \%$, which did not affect Western blot analysis.

Cell culture. Cloned osteoblast-like MC3T3-E1 cells derived from newborn mouse calvaria (19) were maintained as previously described (20). Briefly, the cells were cultured in $\alpha$-minimum essential medium ( $\alpha$-MEM) containing $10 \%$ fetal calf serum (FCS) at $37^{\circ} \mathrm{C}$ in a humidified atmosphere of $5 \%$ $\mathrm{CO}_{2}$ and $95 \%$ air. The cells were seeded into 90-mm diameter dishes in $\alpha$-MEM containing $10 \%$ FCS. After 5 days, the medium was exchanged for $\alpha$-MEM containing $0.3 \%$ FCS. The cells were used for experiments after $48 \mathrm{~h}$. When indicated, the cells were pretreated with Rho kinase inhibitors, Y27632 and fasudil, for $60 \mathrm{~min}$ prior to stimulation of sphingosine 1-phosphate.

Western blot analysis. Western blot analysis was performed as follows. The cultured cells were pretreated with various doses of Y27632 or fasudil for $60 \mathrm{~min}$ and then stimulated by sphingosine 1-phosphate in $\alpha$-MEM containing $0.3 \%$ FCS for the indicated periods. Cells were washed twice with phosphatebuffered saline and then lysed, homogenized and sonicated in a lysis buffer containing $62.5 \mathrm{mM}$ Tris/ $\mathrm{HCl}, \mathrm{pH} 6.8,2 \%$ sodium dodecyl sulfate (SDS), $50 \mathrm{mM}$ dithiothreitol and $10 \%$ glycerol. The cytosolic fraction was collected as a supernatant after centrifugation at $125,000 \mathrm{x}$ g for $10 \mathrm{~min}$ at $4^{\circ} \mathrm{C}$. Cytosolic protein $(20 \mu \mathrm{g})$ was charged, and SDS-polyacrylamide gel electrophoresis (PAGE) was performed according to Laemmli (21) in $10 \%$ polyacrylamide gel. The fractioned proteins were then transferred onto a PVDF membrane (Bio-Rad, Hercules, CA, USA). The protein-transferred membrane was blocked with $5 \%$ fat-free dry milk in Tris-buffered saline-Tween-20 (TBS-T; $20 \mathrm{mM}$ Tris/HCl, pH 7.6, $137 \mathrm{mM} \mathrm{NaCl}, 0.1 \%$ Tween-20) for $2 \mathrm{~h}$ before incubation with the primary anti-
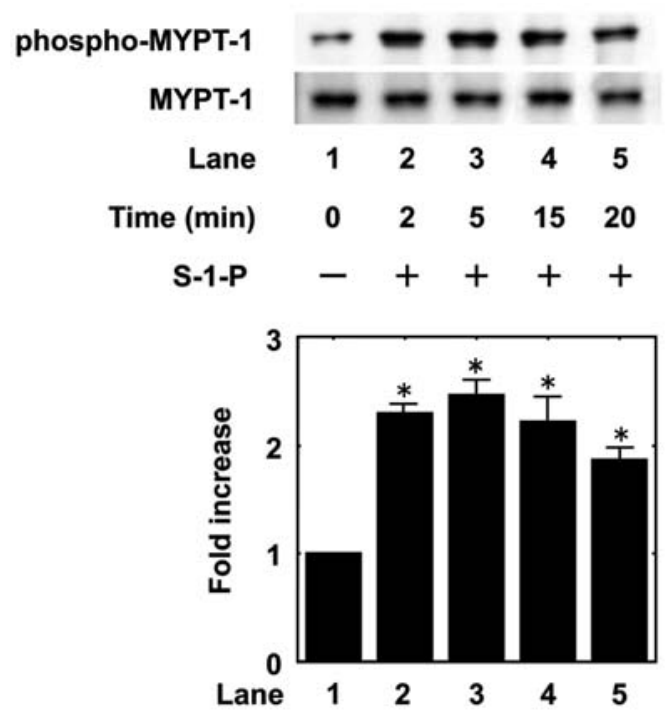

Figure 1. Effects of sphingosine 1-phosphate (S-1-P) on phosphorylation of MYPT-1 in MC3T3-E1 cells. The cultured cells were stimulated with $30 \mu \mathrm{M}$ $\mathrm{S}-1-\mathrm{P}$ for the indicated period. Cell extracts $(20 \mu \mathrm{g})$ were subjected to SDSPAGE with subsequent Western blotting with antibodies against phosphospecific MYPT-1 or MYPT-1. The histogram shows quantitative representations of the levels of S-1-P-induced phosphorylation obtained from laser densitometric analysis of three independent experiments. Each value represents the mean \pm SEM of triplicate determinations. Similar results were obtained with two additional and different cell preparations. The values represent the amount of phospho-specific MYPT-1 divided by those of MYPT-1. ${ }^{*} \mathrm{p}<0.05$, compared to the value of control (lane 1).

bodies. Antibodies for phospho-specific MYPT-1, MYPT-1, HSP27, GAPDH, phospho-specific p38 MAP kinase, p38 MAP kinase, phospho-specific Akt and Akt were used as primary antibodies. Peroxidase-labeled antibodies raised in goat against rabbit IgG (KPL, Inc., Geithersburg, MD, USA) and peroxidase-labeled antibodies raised in rabbit against goat IgG (Abcam, plc. Cambridge, UK) were used as secondary antibodies. The primary and secondary antibodies were diluted at 1:1000 with $5 \%$ fat-free dry milk in TBS-T. Peroxidase activity on PVDF membranes was visualized on X-ray film by means of the ECL Western blotting detection system.

Statistical analysis. The data were analyzed by ANOVA followed by the Bonferroni method for multiple comparisons between pairs and $\mathrm{p}<0.05$ was considered significant. All data are presented as the mean \pm SEM of triplicate determinations. Each experiment was repeated three times with similar results.

\section{Results}

Effects of sphingosine 1-phosphate on the phosphorylation of MYPT-1 in MC3T3-E1 cells. It is generally recognized that MYPT-1, a component of myosin phosphatase, is a downstream substrate of Rho-kinase $(14,22)$. In order to clarify whether sphingosine 1-phosphate activates Rho-kinase in osteoblast-like MC3T3-E1 cells, we examined the effect of sphingosine 1-phosphate on the phosphorylation of MYPT-1. Sphingosine 1-phosphate markedly induced phosphorylation of 
A
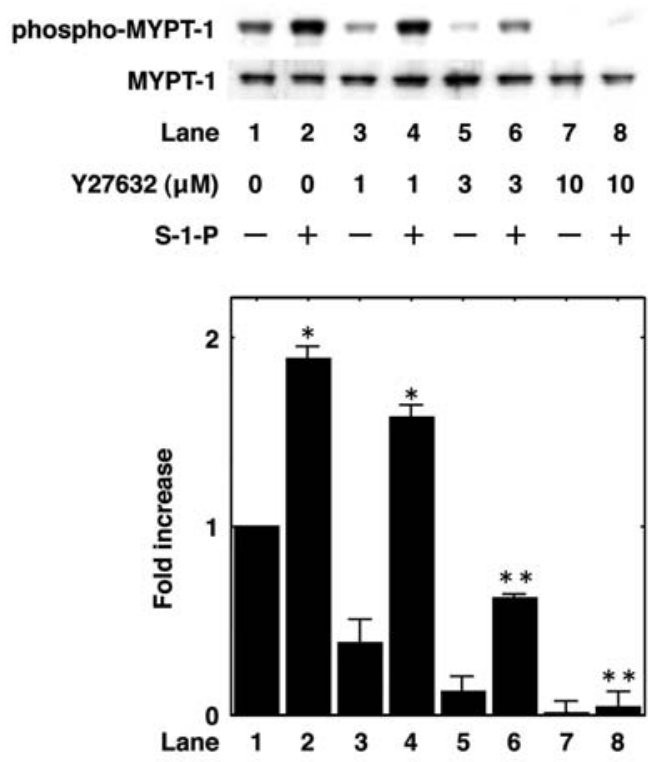

B
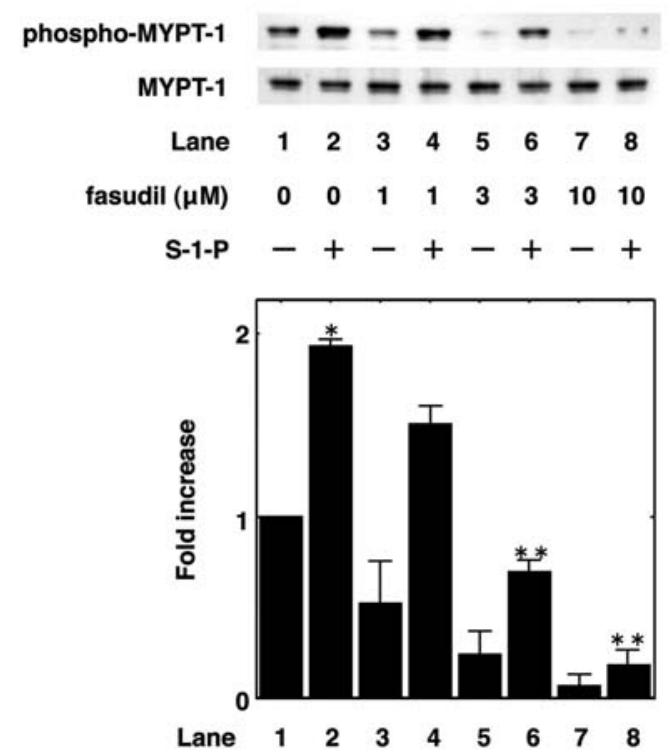

Figure 2. Effects of Y27632 or fasudil on sphingosine 1-phosphate (S-1-P)-induced phosphorylation of MYPT-1 in MC3T3-E1 cells. Cultured cells were pretreated with various doses of Y27632 (A), fasudil (B), or vehicle for $60 \mathrm{~min}$, and then stimulated by $30 \mu \mathrm{M}$ S-1-P or vehicle for 2 min. The histogram shows quantitative representations of the levels of S-1-P-induced phosphorylation obtained from laser densitometric analysis of three independent experiments. Each value represents the mean \pm SEM of triplicate determinations. Similar results were obtained with two additional and different cell preparations. ${ }^{*} \mathrm{p}<0.05$, compared to the control (lane 1 ); ${ }^{* *} \mathrm{p}<0.05$, compared to the value of S-1-P alone (lane 2).

MYPT-1 in a time-dependent manner (Fig. 1). The phosphorylation of MYPT-1 was markedly observed from 2 min after the sphingosine 1-phosphate-stimulation, and still observed 20 min after stimulation (Fig. 1). Y27632, a specific inhibitor of Rho-kinase (16), significantly suppressed sphingosine 1-phosphate-induced phosphorylation levels of MYPT-1 (Fig. 2A). The inhibitory effect on the phosphorylation levels was dose-dependent in the range between 1 and $10 \mu \mathrm{M}$. In addition, fasudil, another inhibitor of Rho-kinase (16), dosedependently reduced sphingosine 1-phosphate-induced levels of MYPT-1 phosphorylation (Fig. 2B).

Effects of Y27632 or fasudil on sphingosine 1-phosphatestimulated induction of HSP27 in MC3T3-E1 cells. We previously showed that sphingosine 1-phosphate stimulates HSP27 induction in osteoblast-like MC3T3-E1 cells $(12,13)$. In order to investigate whether Rho-kinase is involved in the sphingosine 1-phosphate-stimulated induction of HSP27 in these cells, we next examined the effect of Y27632 on the induction of HSP27 by stimulation of sphingosine 1-phosphate. Y27632, which by itself had little effect on the HSP27 levels, significantly suppressed the sphingosine 1-phosphatestimulated induction of HSP27 (Fig. 3A). The inhibitory effect of Y27632 was dose-dependent in the range between 1 and $10 \mu \mathrm{M}$. Y27632 $(10 \mu \mathrm{M})$ completely suppressed the induction of HSP27 stimulated by sphingosine 1-phosphate (Fig. 3A, lane 8). Fasudil, as well as Y27632, reduced the sphingosine 1-phosphate-stimulated induction of HSP27 in these cells (Fig. 3B). The effect of fasudil on the induction of HSP27 was dose-dependent in the range between 1 and $10 \mu \mathrm{M}$. Fasudil
$(10 \mu \mathrm{M})$ caused $\sim 90 \%$ inhibition in the sphingosine 1phosphate effect.

Effects of Y27632 or fasudil on sphingosine 1-phosphateinduced phosphorylation of p38 MAP kinase in MC3T3-E1 cells. In a previous study (12), we demonstrated that p38 MAP kinase acts as a positive regulator in sphingosine 1-phosphatestimulated HSP27 induction. Therefore, we examined the effect of Y27632 on sphingosine 1-phosphate-induced phosphorylation of p38 MAP kinase in MC3T3-E1 cells. Y27632 markedly suppressed sphingosine 1-phosphate-induced phosphorylation of p38 MAP kinase (Fig. 4A). The Y27632effect on the phosphorylation levels was dose-dependent in the range between 1 and $10 \mu \mathrm{M}$. Y27632 $(10 \mu \mathrm{M})$ caused $\sim 80 \%$ inhibition in the sphingosine 1-phosphate-effect. Fasudil reduced sphingosine 1-phosphate-induced levels of phosphorylated-p38 MAP kinase (Fig. 4B). The inhibitory effect of fasudil was dose-dependent in the range between 1 and $10 \mu \mathrm{M}$. Fasudil $(10 \mu \mathrm{M})$ caused almost complete inhibition in the sphingosine 1-phosphate effect.

Effects of Y27632 or fasudil on sphingosine 1-phosphateinduced phosphorylation of Akt in MC3T3-E1 cells. In addition, we showed that independently of p38 MAP kinase activation, sphingosine 1-phosphate induces HSP27 through the PI3K/Akt pathway (13). We next examined the effects of Y27632 and fasudil on sphingosine 1-phosphate-induced phosphorylation of Akt in MC3T3-E1 cells. Even at $10 \mu \mathrm{M}$, both Y27632 and fasudil failed to reduce sphingosine 1phosphate-induced levels of phosphorylated-Akt (Fig. 5). 
A
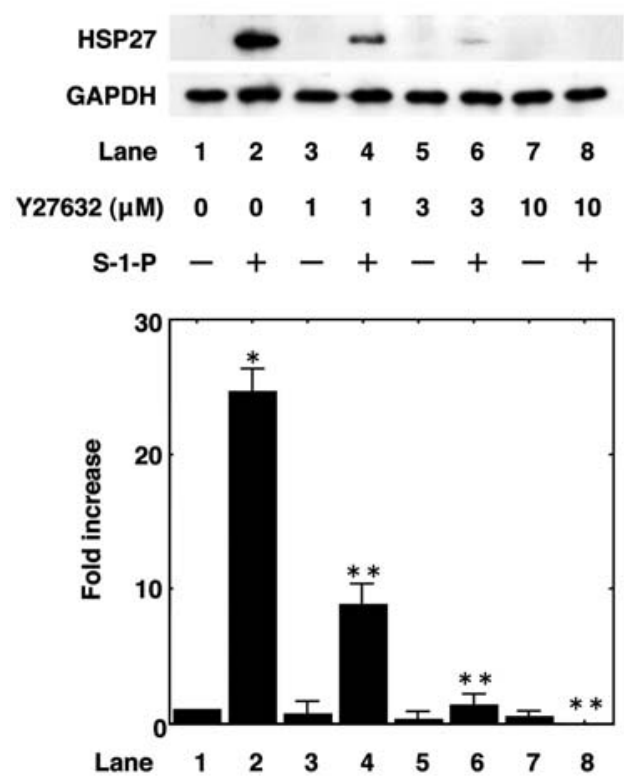

B
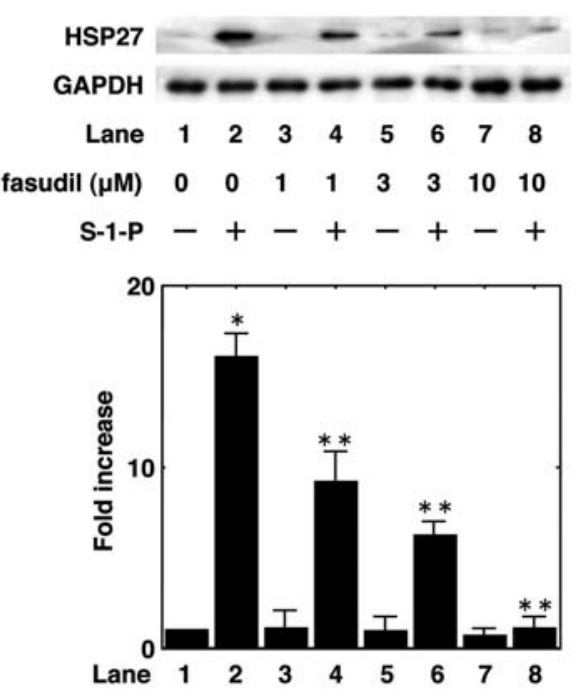

Figure 3. Effects of Y27632 or fasudil on sphingosine 1-phosphate (S-1-P)-induced levels of HSP27 in MC3T3-E1 cells. Cultured cells were pretreated with various doses of Y27632 (A), fasudil (B), or vehicle for $60 \mathrm{~min}$, and then stimulated by $30 \mu \mathrm{M} \mathrm{S}-1-\mathrm{P}$ or vehicle for $8 \mathrm{~h}$. Each value represents the mean \pm SEM of triplicate determinations. Similar results were obtained with two additional and different cell preparations. ${ }^{*} \mathrm{p}<0.05$, compared to the control (lane 1$)$; ${ }^{* *} \mathrm{p}<0.05$, compared to the value of S-1-P alone (lane 2).

A
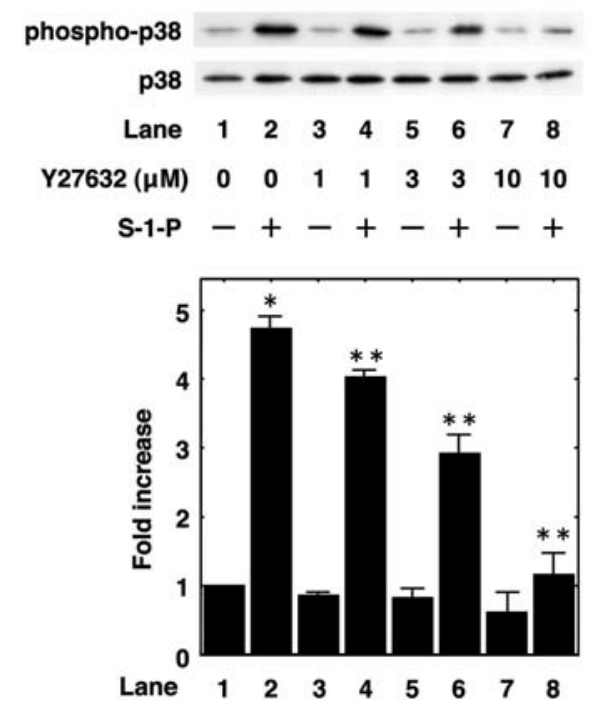

B
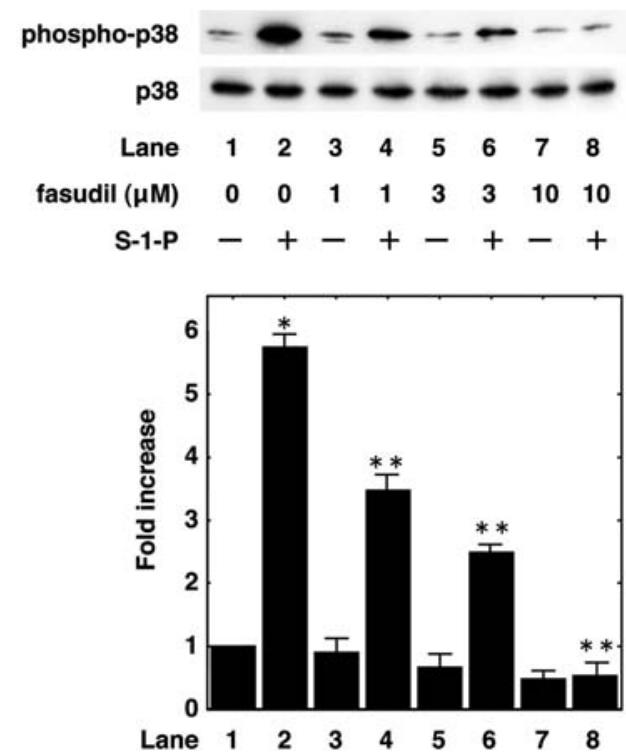

Figure 4. Effects of Y27632 or fasudil on sphingosine 1-phosphate (S-1-P)-induced phosphorylation of p38 MAP kinase in MC3T3-E1 cells. Cultured cells were pretreated with various doses of Y27632 (A), fasudil (B), or vehicle for 60 min, and then stimulated by $30 \mu \mathrm{M}$ S-1-P or vehicle for 10 min. The histogram shows quantitative representations of the levels of S-1-P-induced phosphorylation obtained from laser densitometric analysis of three independent experiments. Each value represents the mean \pm SEM of triplicate determinations. Similar results were obtained with two additional and different cell preparations. ${ }^{*} \mathrm{p}<0.05$, compared to the control (lane 1 ); ${ }^{* *} \mathrm{p}<0.05$, compared to the value of S-1-P alone (lane 2).

\section{Discussion}

We previously showed that sphingosine 1-phosphate stimulates induction of HSP27 in osteoblast-like MC3T3-E1 cells and that p38 MAP kinase takes part in the sphingosine 1-phosphate- effect (12). Furthermore, we recently reported that sphingosine 1-phosphate stimulates the induction of HSP27 via the PI3K/ Akt pathway independently of the p38 MAP kinase pathway in these cells (13). In the present study, we first demonstrated that sphingosine 1-phosphate time-dependently induces the 

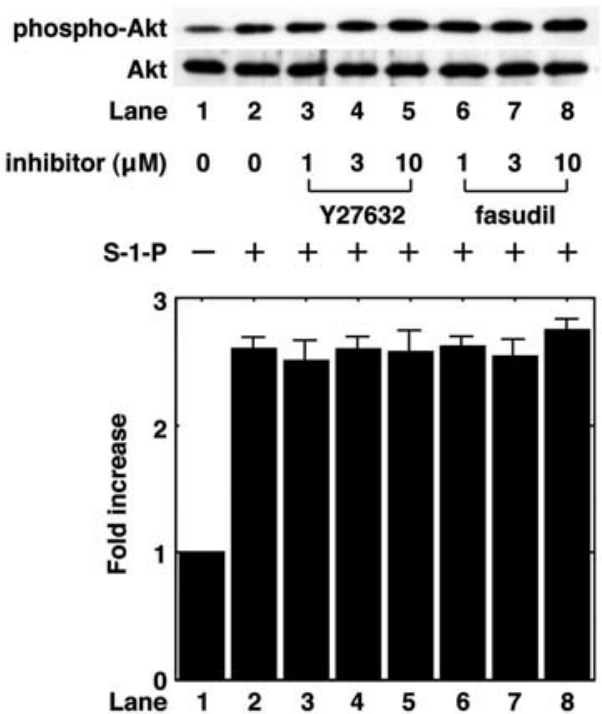

Figure 5. Effects of Y27632 or fasudil on sphingosine 1-phosphate (S-1-P)induced phosphorylation of Akt in MC3T3-E1 cells. Cultured cells were pretreated with various doses of Y27632 (lane 3-5), fasudil (lane 6-8) or vehicle (lanes 1 and 2) and then stimulated by $30 \mu \mathrm{M} \mathrm{S}-1-\mathrm{P}$ or vehicle for $15 \mathrm{~min}$. The histogram shows quantitative representations of the levels of $\mathrm{S}$-1-P-induced phosphorylation obtained from laser densitometric analysis of three independent experiments. Each value represents the mean \pm SEM of triplicate determinations. Similar results were obtained with two additional and different cell preparations. ${ }^{*} \mathrm{p}<0.05$, compared to the control (lane 1 ).

phosphorylation of MYPT-1 in osteoblast-like MC3T3-E1 cells, using phospho-specific MYPT-1 (Thr850) antibodies. MYPT-1, which is a myosin-binding subunit of myosin phosphatase and regulates the interaction of actin and myosin, is well known as a downstream target of Rho-kinase $(14,22)$. In addition, we showed that Y27632 and fasudil, specific inhibitors of Rho-kinase (16), suppressed the sphingosine 1phosphate-induced phosphorylation of MYPT-1. Therefore, our results indicate that sphingosine 1-phosphate elicits activation of Rho-kinase in osteoblast-like MC3T3-E1 cells.

In bone metabolism, it was reported that the activation of Rho-kinase suppresses the differentiation of osteoblasts and induces their proliferation (17). Recently, we showed that Rho-kinase in osteoblasts acts as a positive regulator in the synthesis of IL- 6 , one of the central modulators of bone metabolism (18). Therefore, the Rho-kinase pathway in osteoblasts is a new candidate for a molecular therapeutic target of fracture healing, bone formation and bone metabolic disease, such as osteoporosis. However, the exact role of Rho-kinase activation in osteoblasts is not fully understood. We previously reported that the activation of the p38 MAP kinase and PI3K/Akt pathways are involved in HSP27 induction by sphingosine 1-phosphate in osteoblast-like MC3T3-E1 cells $(12,13)$. Therefore, we next investigated the involvement of Rho-kinase in the sphingosine 1-phosphate-stimulated HSP27 induction in MC3T3-E1 cells. In the present study, sphingosine 1-phosphate-stimulated HSP27 induction was reduced by Rhokinase inhibitors, Y27632 and fasudil. In addition, Rho-kinase inhibitors suppressed sphingosine 1-phosphate-induced phosphorylation of p38 MAP kinase. However, neither inhibitor attenuated the sphingosine 1-phosphate induced-phosphorylation levels of Akt. These findings suggest that Rhokinase plays an important role at a point upstream of not Akt

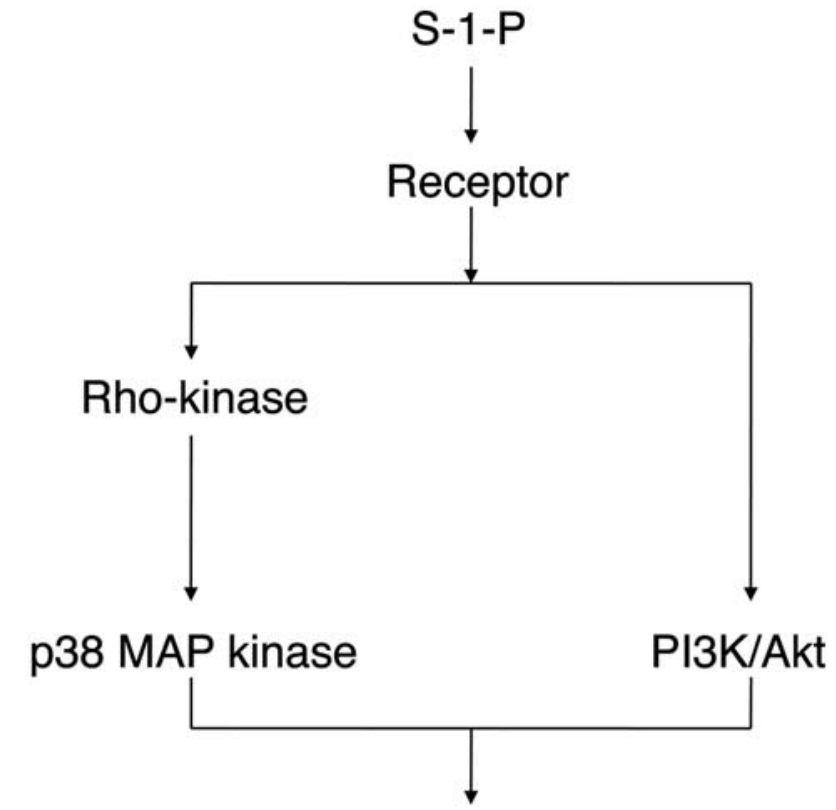

\section{HSP27 induction}

Figure 6. Schematic representation of the involvement of Rho-kinase in the mechanism of sphingosine 1-phosphate (S-1-P)-stimulated HSP27 induction in osteoblast-like MC3T3-E1 cells.

but p38 MAP kinase in the sphingosine 1-phosphate-stimulated HSP27 induction in osteoblast-like MC3T3-E1 cells. It is probable that the Rho-kinase pathway functions independently of the PI3K/Akt pathway in HSP27 induction. It was reported that MC3T3-E1 cells express the endothelial differentiation genes (Edg)-1 and -5 (23), plasma membrane receptors for sphingosine 1-phosphate mediating its extracellular stimulation $(24,25)$. The potential mechanism of the sphingosine 1phosphate-stimulated HSP27 induction shown herein is summarized in Fig. 6.

Sphingosine 1-phosphate reportedly prevents apoptosis in primary rat osteoblasts and human osteosarcoma SaOS-2 cells (26). It was shown that estrogen-induced resistance to osteoblast apoptosis is associated with increased HSP27 expression (27). Furthermore, it was reported that activation of Rho-kinase suppresses differentiation of osteoblasts and induces their proliferation (17). Although the physiological significance of HSP27 in osteoblasts is not yet clarified, the expression of HSP27 via the Rho-kinase/p38 MAP kinase pathway in osteoblasts might be related to the maintenance of the number of viable osteoblasts in bone tissue. Further investigations are necessary to clarify the exact role of HSP27 in osteoblasts. Taken together, our results strongly suggest that Rho-kinase is involved in the sphingosine 1-phosphatestimulated induction of HSP27 via p38 MAP kinase in osteoblasts.

\section{Acknowledgements}

We are very grateful to Yoko Kawamura for her skillful technical assistance. This investigation was supported in part by Grant-in-Aid for Scientific Research (19591042) from the Ministry of Education, Science, Sports and Culture of Japan, the Foundation for Growth Science, the Research Grants for 
Longevity Sciences (15A-1 and 15C-2), Research Grant on Proteomics and Research Grant on Longevity Sciences from the Ministry of Health, Labour and Welfare of Japan.

\section{References}

1. Spiegel S and Merrill JAH: Sphingolipid metabolism and cell growth regulation. FASEB J 10: 1388-1397, 1996.

2. Spiegel S and Milstein S: Sphingosine-1-phosphate: An enigmatic signaling lipid. Nat Rev Mol Cell Biol 4: 397-407, 2003.

3. Sanchez T and Hla T: Structural and functional characteristics of SIP receptors. J Biol Chem 92: 913-922, 2004.

4. Kozawa O, Tokuda $\mathrm{H}$, Matsuno $\mathrm{H}$ and Uematsu T: Activation of mitogen-activated protein kinase is involved in sphingosine 1-phosphate-stimulated interleukin-6 synthesis in osteoblasts. FEBS Lett 418: 149-151, 1997.

5. Hendrick JP and Hartl FU: Molecular chaperone functions of heat-shock proteins. Annu Rev Biochem 62: 349-384, 1993.

6. Inaguma Y, Goto S, Shinohara H, Hasegawa K, Ohshima K and Kato K: Physiological and pathological changes in levels of the two small stress proteins, HSP27 and alpha B crystallin, in ra hindlimb muscles. J Biol Chem (Tokyo) 114: 378-384, 1993.

7. Benjamin IJ and McMillan DR: Stress (heat shock) proteins: Molecular chaperones in cardiovascular biology and disease. Circ Res 83: 117-132, 1998.

8. Gaestel M, Schroder W, Benndorf R, Lippmann C, Bunchner K Hucho F, Erdmann VA and Bielka H: Identification of the phosphorylation sites of the murine small heat shock protein hsp25. J Biol Chem 266: 14721-14724, 1991.

9. Landry J, Lambert H, Zhou M, Lavoie JN, Hickey E, Weber LA and Anderson CW: Human HSP27 is phosphorylated at serines 78 and 82 by heat shock and mitogen-activated kinases that recognize the same amino acid motif as S6 kinase II. J Biol Chem 267: 794-803, 1992.

10. Kato K, Hasegawa K, Goto S and Inagma Y: Dissociation as a result of phosphorylation of an aggregated form of the small stress protein, hsp27. J Biol Chem 269: 11274-11278, 1994.

11. Rogalla T, Ehrnsperger M, Preville X, Kotlyarov A, Lutsch G, Ducasse C, Paul C, Wieske M, Arrigo AP, Buchner J and Gaestel M: Regulation of Hsp27 oligomerization, chaperone function, and protective activity against oxidative stress/tumor necrosis factor $\alpha$ by phosphorylation. J Biol Chem 274: 18947-18956, 1999

12. Kozawa O, Niwa M, Matsuno $H$, Tokuda $H$, Miwa M, Ito $H$, Kato $\mathrm{K}$ and Uematsu T: Sphingosine 1-phosphate induces heat shock protein 27 via p38 mitogen-activated protein kinase activation in osteoblasts. J Bone Miner Res 92: 1203-1211, 1999.

13. Takai S, Tokuda H, Matsushima-Nishiwaki R, Hanai Y, Kato K and Kozawa O: Phosphatidylinositol 3-kinase/Akt plays a role in sphingosine 1-phosphate-stimulated HSP27 induction in osteoblasts. J Cell Biochem 98: 1249-1256, 2006.
14. Fukata Y, Amano M and Kaibuchi K: Rho-Rho-kinase pathway in smooth muscle contraction and cytoskeletal reorganization of non-muscle cells. Trends Pharmacol Sci 22: 32-39, 2001.

15. Riento K and Ridley AJ: Rocks: multifunctional kinases in cell behavior. Nat Rev Mol Cell Biol 4: 446-456, 2003.

16. Shimokawa H and Rashid M: Development of Rho-kinase inhibitors for cardiovascular medicine. Trends Pharmacol Sci 28: 296-302, 2007.

17. Harmey D, Stenbeck G, Nobes CD, Lax AJ and Grigoriadis AE: Regulation of osteoblast differentiation by Pasteurella multocida toxin (PMT): a role for Rho GTPase in bone formation. J Bone Miner Res 19: 661-670, 2004.

18. Minamitani C, Otsuka T, Takai S, Matsushima-Nishiwaki R, Adachi S, Hanai Y, Mizutani J, Tokuda $\mathrm{H}$ and Kozawa O: Involvement of Rho-kinase in prostaglandin F2 $\alpha$-stimulated interleukin-6 synthesis via p38 mitogen-activated protein kinase in osteoblasts. Mol Cell Endocrinol 291: 27-32, 2008.

19. Sudo H, Kodama H, Amagai Y, Yamamoto S and Kasai S: In vitro differentiation and calcification in a new clonal osteogenic cell line derived from newborn mouse calvaria. J Cell Bio 196: 191-198, 1983.

20. Kozawa O, Suzuki A, Tokuda H and Uematsu T: Prostaglandin F2 $\alpha$ stimulates interleukin-6 via activation of PKC in osteoblastlike cells. Am J Physiol 272: E208-E211, 1997.

21. Laemmli UK: Cleavage of structural proteins during the assembly of the head of bacteriophage T4. Nature 227: 680-685, 1970.

22. Ito M, Nakano T, Erdodi F and Hartshorne DJ: Myosin phosphatase: structure, regulation and function. Mol Cell Biochem 259: 197-209, 2004.

23. Lyons JM and Karin N: A role for G protein-coupled lysophospholipid receptors in sphingolipid-induced $\mathrm{Ca}^{2+}$ signaling in MC3T3-E1 osteoblastic cells. J Bone Miner Res 16: 2035-2042, 2001.

24. Lee MJ, Van Brocklyn JR, Thangada S, Liu CH, Hand AR, Menzeleev R, Spiegel S and Hla T: Sphingosine-1-phosphate as a ligand for the $\mathrm{G}$ protein-coupled receptor EDG-1. Science 279: 1552-1555, 1998.

25. Zondag GCM, Postma FR, van Etten I, Verlaan I and Moolenaar WH: Sphingosine 1-phosphate signaling through the G-protein-coupled receptor Edg-1. Biochem J 330: 605-609, 1998.

26. Grey A, Chen Q, Callon K, Xu X, Reid IR and Cornish J: The phospholipids sphingosine 1-phosphate and lysophosphatidic acid prevent apoptosis in osteoblastic cells via a signaling pathway involving Gi proteins and phosphatidylinositol-3 kinases. Endocrinology 143: 4755-4763, 2002.

27. Cooper LF, Tiffee JC, Griffin JP, Hamano H and Guo Z: Estrogen-induced resistance to osteoblast apoptosis is associated with increased hsp27 expression. J Cell Physiol 185: 401-407, 2000 . 